\title{
INDICATORS OF BODY RESERVE OPPORTUNITIES FOR YOUNG GYMNASTS AS ONE OF THE FACTORS FOR SUCCESSFUL DEVELOPMENT OF INITIAL SPECIALIZED TRAINING
}

\author{
Zinaida Kuznetsova, Ilshat Dauletshin, Stanislav Kuznetsov \\ Povolzhskaya State Academy of Physical Culture, Sport and Tourism, \\ Naberezhnye Chelny, Russia
}

\begin{abstract}
Background. The article deals with the problem of selection in gymnastics and the reasons why it is necessary to research that. Artistic gymnastics carries the heaviest losses on the initial stages of specialized training, which explains the lack of science-based regulatory requirements in the gymnastics system, allowing diagnosing, assessing and predicting the development of the main indicators of physical condition and fitness of athletes in complex.

Research aim was to develop additional criteria of young gymnasts' selection at the initial stage of specialized training on the basis of the indicators of human body reserve opportunities.

Methods. Experimental work was carried out with gymnasts aged 9 years $(n=15)$ and 10 years $(n=16)$ in the research laboratory of Povolzhskaya State Academy of Physical Culture, Sport and Tourism and sports complex "Yar Chally" in Naberezhnye Chelny, Republic of Tatarstan (Russia).

Results. Gymnasts aged 9-10 years had a harmonious type of power supply of the body: more gymnasts had aerobic-anaerobic type of muscular activity power supply. Gymnasts who qualified in the obligatory program of category 3 on pommel horse equally represented only the second and the third biological groups.

The results of correlation analysis suggest that for gymnasts aged 9 years, the most significant factors affecting their special physical and technical fitness included aerobic capacity source of muscle activity energy supply and general metabolic capacity and for gymnasts aged 10 years - capacity and anaerobic power glycolytic sources of energy and muscular activity.

Discussion and conclusions. Obtained information on the preferred for gymnastics bioenergy groups can help coaches in predicting abilities of young gymnasts.
\end{abstract}

Keywords: sportspeople, gymnasts, selection, model descriptions, indicators of reserve opportunities.

\section{INTRODUCTION}

$\mathrm{T}$

The problem of the scientific substantiation of selection and orientation in sports has always existed in our country and abroad (Melikhova, 2007; Rozin \& Mukambetov, 1980; Rozin, 2001; Shlemin \& Bril, 1978; Smolevsky, 2005). The solution of this problem allows reducing the time of high-class athlete training, giving them thus a longer period for performance in the international arena (Lebedev \& Rozin, 1981; Melikhova, 2007; Vasilchuk \& Gaverdovskiy, 1985).

Prevailing training and technology in Russian gymnastics should be improved in line with the trends of gymnastics (Arkaev \& Rozin, 1999). It should be noted that this provision applies fully to the technology of selection and sports orientation in the gym. We suggest the following main reasons 
for the need to improve methods of selection and sports orientation of young gymnasts.

First, at present almost all competitions in gymnastics among children are conducted following the mandatory programs approved by the Russian Federation of Gymnastics, which is a set of required elements for each type of all-around gymnastics and gymnasts (Grigoryants, 2005).

Secondly, the recently adopted rules allow gymnasts to compete specializing in individual events (Green, 2006; Kirk Seeley \& Bressel, 2005; Marinšek \& Čuk, 2010). Results of the 2004 Olympic Games in Athens showed that most successful among men were gymnasts-"specialists". Smolevsky (2005) claims that the rules can be criticized from the standpoint of events and overall achievements of prestigious teams, but among the winners there were athletes over 25 years of age, and the gold medals for men were won by representatives from eight out of eight different countries. According to the scientist, position of the International Gymnastics Federation (FIG) is unlikely to change in the foreseeable future and this fact should be considered when developing a strategy and tactics for gymnasts' long-term training.

Aforementioned facts prove the relevance of the problem and the need for additional selection criteria in gymnastics.

Research aim was to develop additional criteria of young gymnasts' selection at the initial stage of specialized training on the basis of the indicators of the body reserve opportunities.

Research objectives were as follows:

1. To study the indicators of body reserve opportunities for gymnasts qualified in junior categories I and III.

2. To identify the features of body reserve opportunities typical of young gymnasts.

\section{METHODS}

Methods of research chosen were theoretical analysis and synthesis of research literature, pedagogical experiment, medical and biological methods (computer technique "D\&K-TEST"), testing of specific physical (SPF) and technical fitness (STF), and methods of mathematicalstatistical data processing.

Experimental work was carried out with gymnasts aged 9 years $(n=15)$ and 10 years $(n=16)$ in the research laboratory of Povolzhskaya State Academy of Physical Culture, Sport and Tourism and sports complex "Yar Chally" Naberezhnye Chelny, Republic of Tatarstan, Russia.

Computer technique "D\&K-TEST" was used to analyse the following indicators: the ANAMV anaerobic metabolic volume; AMV - aerobic metabolic volume; GMV - general metabolic volume; $\mathrm{PCP}$ - the power creatine phosphate source of energy supply; PGL - the power glycolytic source of energy supply; MASES - the power aerobic source of energy supply (maximum oxygen consumption); W ANMT - anaerobic metabolism threshold; heart rate ANMT - criterion of efficiency of the use of aerobic source.

The percentage of the ANAMV and AMV indicates five bioenergy types: aerobic type of power supply for muscular activity, aerobicglycolytic type of power supply for muscular activity, aerobic-anaerobic (mixed) energy supply for the optimal level of muscle activitiy, anaerobicaerobic type with a high level of anaerobic energy for muscle activity, anaerobic type with the highest level of anaerobic energy for muscle activity.

The first group includes athletes with ANAMV (1-11\%) and AMV (89-99\%), the second group with ANAMV (12-20\%) and AMV (80-88 \%), the third group - with ANAMV (21-29\%) and AMV (71-79\%), the fourth group includes athletes with ANAMV (30-38\%) and AMV (62-70\%) and to the fifth group - athletes with ANAMV (39-83\%) and AMV (17-61\%) (Dushanin, 1986; Gibadullin, 2009; V. P Karlenko \& N. V. Karlenko, 2003).

\section{RESULTS}

The study of reserve opportunities in gymnasts' bodies revealed that among 9-year-olds, 20\% of them belong to the second bioenergy group with a predisposition for aerobic work, $66.7 \%$ belong to the third bioenergy group with a predisposition equal to both aerobic and anaerobic work, and $13.3 \%$ belong to the fourth bioenergy group with a predisposition to anaerobic work. As for 10 -yearold gymnasts, $25 \%$ of them belong to the second group, $56.2 \%$ - the third group, and $18.8 \%$ - the fourth group. Summarizing the data for all ages, we identified $22.6 \%$ of the research participants who belong to the second group, $61.3 \%$ of them in the third group, and $16.1 \%$ - in the fourth group. Among the young gymnasts, there were no representatives in the first and the fifth biogroups (Figure 1). 
Table. Indicators $(\mathbf{X} \pm \boldsymbol{\sigma})$ of body reserve opportunities for young gymnasts

\begin{tabular}{|c|c|c|c|c|c|c|c|c|}
\hline Age & ANAMV & AMV & GMV & PCP & PGL & MASES & W ANMT & $\begin{array}{c}\text { Heart rate } \\
\text { ANMT }\end{array}$ \\
\hline \multirow{2}{*}{ 9 years } & $89.8 \pm$ & $268.9 \pm$ & $358.7 \pm$ & $29.1 \pm$ & $35.8 \pm$ & $75.0 \pm$ & $67.7 \pm$ & $178.6 \pm$ \\
& 20.6 & 18.7 & 17.7 & 5.5 & 4.3 & 3.9 & 3.0 & 5.2 \\
\hline \multirow{2}{*}{10 years } & $75.4 \pm$ & $246.5 \pm$ & $321.9 \pm$ & $30.9 \pm$ & $31.8 \pm$ & $69.8 \pm$ & $68.9 \pm$ & $170.6 \pm$ \\
& 18.5 & 23.7 & 28.1 & 3.9 & 7.5 & 4.1 & 5.0 & 5.2 \\
\hline
\end{tabular}

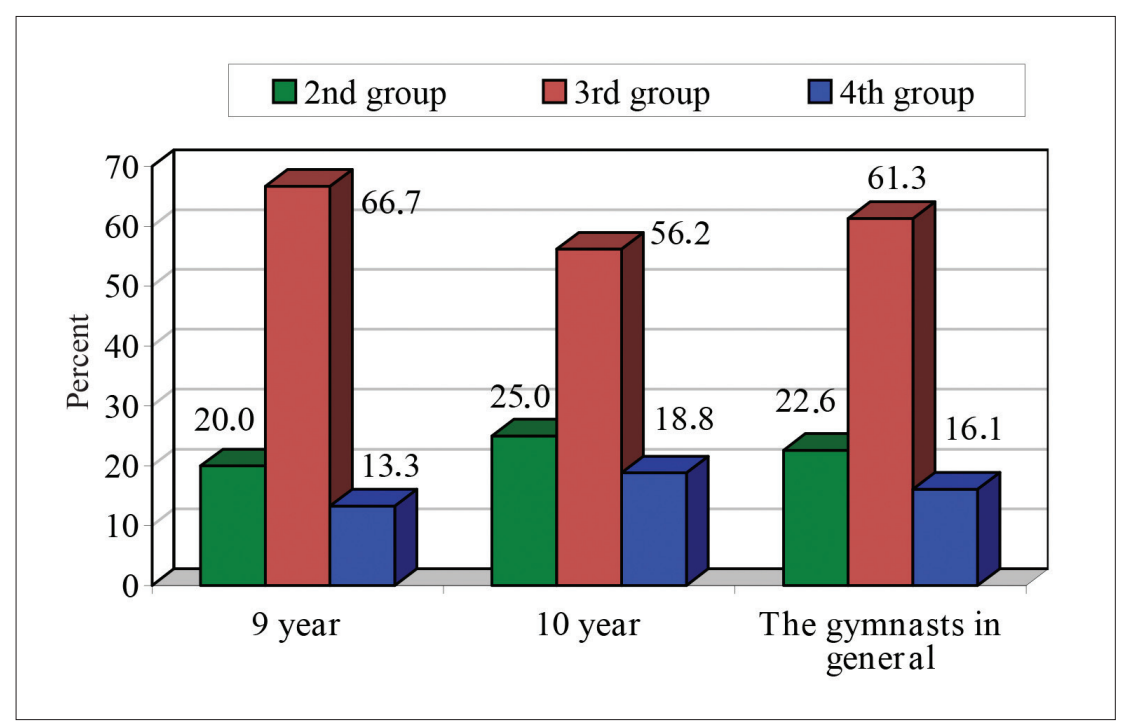

Figure. The distribution of bioenergy groups among 9-10-year-old gymnasts

Our research data obtained indicate that 9-yearold gymnasts in the third biogroup can more easily meet the special requirements for the types of allaround gymnastics. Among the 10 -year-old gymnasts in all kinds of Multi-Athlon, except exercises on pommel horse, there is a dominance of the third biogroup compared to the second biogroup. Gymnasts who were in compliance with the mandatory requirements of the program on the pommel horse equally represented the second and the third biogroups.

The total analysis of the data for all ages showed that among the young gymnasts mandatory program for all kinds of gymnastics was more accessible by members in the third biogroup.

Next, we consider the results of the correlation analysis between indicators of body reserve opportunities and SPP and STP indicators among the gymnasts aged 9-10 years.

Among 9-year-old gymnasts, reliable correlation values were found in the following pairs:

- ANAMV in combination with the results of the test "circles" $(r=-.66)$;

- AMV in combination with "Swiss press to handstand" test results $(r=-.63)$;
- GMV in combination with "Swiss press to handstand" test results $(r=-.54)$, "hanging scale rearways (back lever)" ( $r=-.62)$, "circles" ( $r=-.63)$; "handstand" $(r=.67)$, STP on the pommel horse $(r=-.58)$;

- MASES in combination with "climbing on a rope" test results $(r=.77)$, "V-sit" $(r=-.60)$, "Swiss press to handstand" $(r=-.66)$, "lifting force" $(r=-.52)$, "hanging scale rearways (back lever)" ( $r=-.75)$, "handstand" $(r=-.57)$.

For 10-year-old gymnasts, significant correlation values were found in the following pairs:

- ANAMV in combination with the "jump" test results $(r=-.51)$, "climbing on a rope" ( $r=.62)$, "V-sit" ( $r=-.68)$, "Swiss press to handstand" ( $r=-.54)$, "lifting force" $(r=-.60)$, "hanging scale rearways (back lever)" ( $r=.89)$, "circles" $(r=-.56)$, "handstand" $(r=-.66)$, STP on the floor exercises $(r=.59)$, pommel horse $(r=-.54)$, rings $(r=-.65)$, parallel bars $(r=-.66)$, the horizontal bar $(r=-.65)$, and total STP $(r=-.69)$; 
- AM, combined with the results of the test "20 m run" $(r=-.57)$;

- PCP combined with the "Swiss press to handstand" test results $(r=-.53)$;

- PGL in combination with the results of the tests "20 m run" $(r=.53)$, "Swiss press to handstand" $(r=-.56)$, "hanging scale rearways (back lever)" $(r=-.56)$, "handstand" $(r=-.56)$, STP on the floor exercises $(r=-.56)$, horizontal bar $(r=.61)$ and total $\operatorname{STP}(r=-.52)$;

- W ANMT in combination with the results of the tests "20 m run" ( $r=-.56)$, "handstand" $(r=.53)$, STP on the floor exercises $(r=.55)$ and horizontal bar $(r=.60)$.

\section{DISCUSSION}

Numerous studies of the authors (Dushanin, 1986; Gibadullin, 2009; V. P. Karlenko \& N. V. Karlenko, 2003) allowed us to develop a new method for determining the functional capacity of aerobic and anaerobic components without the use of load tests. It was found that between the permeability of cell membranes of the myocardium, the slew rate of the transmembrane action potential in ventricular depolarization of the heart was recorded in a state of rest and the values of aerobic and anaerobic metabolism - in muscle activity of any orientation, length and intensity.

The basis for quick diagnosis of chest electrocardiogram in such leads as $\mathrm{V}_{3 \mathrm{R}}, \mathrm{V}_{1}$ and $\mathrm{V}_{2}$, $\mathrm{V}_{4}, \mathrm{~V}_{5}$, and $\mathrm{V}_{6}$ in muscle at rest is registered. In each of them, R-wave amplitude (in $\mathrm{mm}$ ) and $\mathrm{S}$ are measured and their percentages $(\mathrm{R} \times 100) /(\mathrm{R}+$ $S)$ which allow defining various aspects of athlete functional performance are calculated.

This method of quick diagnosis enables to determine various aspects of functional and reserve capacity of athletes' bodies without complicated procedures and to keep records and control of the current operational status of athletes. During the diagnosis and mathematical calculations of adjustment of $\mathrm{R}$ and $\mathrm{S}$ electrocardiogram waves, researchers (Dushanin, 1986; V. P. Karlenko \& N. V. Karlenko, 2003) have identified various functional parameters:

- The general metabolic capacity (GMV) shows a certain amount of aerobic (AMV) and anaerobic (ANAMV) metabolic volumes during muscular work with the intensity level of maximum oxygen consumption.
The indicator shows a certain level of performance.

- Power creatine phosphate source of energy (PCP) is the fastest source of energy used by muscle cells from the beginning of muscle contraction. It occurs due to the high-energy phosphate compounds - creatine phosphate. This source of energy is characterized by the maximum possible rate movements. An athlete cannot perform such movements for more than 10 seconds. The creatine phosphate source has a maximum capacity in comparison with other systems. Maximum speed of energy production exceeds the capacity of the glycolytic system three times and the aerobic capacity of the system 4-10 times. Capacity creatine phosphate system is small and many times less than other systems. In the operation of this power, request of the total oxygen is small, but oxygen qcquisition time is significantly longer. Therefore, the work in this capacity is also anaerobic because re-synthesis of ATP oxygen is not involved. These indicators of power creatine phosphate source of energy prove the effectiveness of the use of tools and methods in the training process in the development of power as well as explosive speed and power abilities (Sandbakk, Holmberg, \& Leirdal, 2010).

- The power glycolytic energy source (PGL) is characterized by anaerobic degradation reaction of glycogen. As a result of such reactions, lactic acid - lactate is formed. The totality of these reactions is called anaerobic glycolysis. This system is characterized by high energy capacity, i.e. a high rate of energy production. Power anaerobic-glycolytic energy source reaches a maximum only at work that lasts longer than 10-20 seconds. This system plays a crucial role in energy supply works very large capacity, which can last from 10 seconds to $2-3$ minutes and is associated with strong muscle contractions. Power-anaerobic glycolytic power 1,5 times higher than that of aerobic, but 3 times lower than power creatine phosphate source. However, if to compare data capacity systems, the capacity of the glycolytic source is still above creatine phosphate source which is 2.5 times higher. The indicator assesses the effectiveness of the use of means and 
methods for a certain period of training in the development of speed endurance.

- The power of aerobic energy source (MASES) depends on the level of maximal oxygen consumption (MOC) in the human organism as a whole. Maximum oxygen consumption is an indicator of aerobic performance of the body which is able to perform heavy work providing energy costs due to oxygen absorbed during operation. Therefore, in exercise endurance athletic performance is largely determined by aerobic capacity of an athlete that is largely dependent on the level of maximum oxygen consumption. This indicator is an expression of the community of many body systems (respiratory, cardiovascular, circulatory), and depends on factors such as partial pressure of oxygen in the inhaled air, ventilation, the rate of diffusion of gases from the lungs into the blood, the oxygen capacity of the blood, the volumerate offlow, arterial-venousdifference, and the activity of oxidative enzymes (Mahood, Kenefick, Kertzer, \& Quinn, 2001; Wagner, 1996).

- Efficient and economical use of aerobic source of energy is determined by indicators such as the threshold of anaerobic metabolism (W ANMT) and heart rate at the level of the threshold of anaerobic metabolism (heart rate ANMT). Anaerobic threshold characterizes the level of fitness of the body and the relationship between aerobic and anaerobic energy pathways of exercise. Its anaerobic threshold determines the quality and efficiency of aerobic system. Therefore, the higher the anaerobic threshold, the higher the fitness of the athlete is. The most informative determinant of the anaerobic threshold is heart rate ANMT. That is, the heart rate of the anaerobic threshold demonstrates the level of the aerobic capacity of athletes involved in cyclic sports. Most authors consider heart rate ANMT as most effective in the evaluation and planning of the intensity of training loads as it can be determined by heart rate ANMT transition boundary between aerobic and anaerobic processes. The higher the heart rate ANMT, the higher training intensity can be maintained according to the athlete. Therefore, knowing heart rate ANMT can most effectively allocate zone intensity training loads (Kachouri, Vandewalle, \& Huet, 1996).

On the basis of numerous studies on this procedure, Dushanin (1986), V. P. Karlenko and N. V. Karlenko (2003) concluded that aerobic and anaerobic organism genotypes of athletes of all ages and specialties virtually are unchanged for a long time and are determined by metabolizer genotype. The results of years of research by the method of fast diagnosis "D\&K-TEST" practice in training athletes showed that most high results were achieved by athletes who belonged to the fifth and the fourth bioenergy groups. Their best achievements were shown in individual programs. High achieving first, second and third groups are primarily part of the functional declines and breakdowns in crucial moments according to the statostics.

According to test results of "D\&K-TEST", researchers (Gibadullin, 2009; Gibadullin \& Kozhevnikov, 2010; Gizatullina, 2013; Khalikov, 2014; Kozhevnikov, 2010, 2011; Kugaevsky, 2009; Morozov, 2014) provided recommendations on the structure and content of sports training, on the basis of bioenergy groups of athletes. For the first bioenergy group, the authors recommended the optimal ratio of exercise on the stages of sports perfection and the annual cycle in the zones of intensity which were $90-95 \%$ (zones 1-3), and $5-10 \%$ (zones 4-5). For the second bioenergy group, the intensity of $75-80 \%$ in zones $1-3$ and $20-25 \%$ in zones $4-5$ was recommended. Athletes of the third bioenergy group were recommended the intensity of $70-75 \%$ in zones $1-3$ and $25-30 \%$ in zones 4-5. The fourth bioenergy group was recommended the ratio of the intensity of $65-70 \%$ in zones $1-3$ and $30-35 \%$ in zones $4-5$. Athletes of the fifth group were recommended the intensity of $60-65 \%$ in zones $1-3$ and $35-40 \%$ in the zones $4-5$.

The selection system is shown as one of the contradictions of modern gymnastics: the struggle for mass sports, bringing to sport a number of studies dealing with them and, along with it, the professional selection of promising gifted athletes in the inevitable classifying contingent (Chernukhina, 2004; Čuk \& Marinšek, 2013; Dauletshin, 2013; Rozin, 2001). Rozin (2001) emphasizes that gymnastics carries the heaviest losses in the initial stages of specialized training, which explains the lack of scientific-based regulatory requirements in the gymnastics system allowing diagnosing, assessing and predicting the development of the main indicators of physical condition and fitness of athletes in complex. 
Gibadullin and Kozhevnikov (2010) emphasize that the existing methods of selection and control of the training process are the most efficient methods of Dushanin and Karlenko in the "Assessment and functional reserve capacity of the organism" "D\&K-TEST". Further research is needed in this direction.

\section{CONCLUSIONS}

1. Gymnasts aged 9-10 years have a harmonious type of power supply to the body: more gymnasts have aerobic-anaerobic type of muscular activity power supply. Gymnasts who qualified in the obligatory program in category 3 on pommel horse equally represented only the second and the third biological groups.

2. The results of correlation analysis suggest that gymnasts aged 9 years employ the aerobic capacity source of muscle activity energy supply and general metabolic capacity, and gymnasts aged 10 years - the capacity and anaerobic power glycolytic sources of energy for their muscular activity.

The information obtained on the bioenergy groups preferred in gymnastics can help coaches in predicting the abilities of young gymnasts. Further research is needed in this direction.

\section{REFERENCES}

Arkaev, L. Y., \& Rozin, E. Y. (1999). Age, height and weight indices, mastery of gymnasts-juniors. Theory and Practice of Physical Culture, 5, 47-51.

Chernukhina, O. V. (2004). Tackling preserving children in sport: A case of women's gymnastics. Physical Culture: Upbringing, Education and Training, 1, 37-38. Čuk, I., \& Marinšek, M. (2013). Landing quality in artistic gymnastics is related to landing symmetry. Biology of Sport, 30(1), 29-33. doi: 10.5604/20831862.1029818

Dauletshin, I. I. (2013). Description of the physical fitness of 7-year-old children on the stage of selection for going in for artistic gymnastics. PedagogicalPsychological and Medico-Biological Problems of Physical Culture and Sports, 1(26), 31-39.

Dushanin, S. A. (1986). System multifactor rapid diagnosis of functional readiness of athletes at the current operational and medical-pedagogical control. Kiev: Intermed.

Gibadullin, I. G., \& Kozhevnikov, V. S. (2010). Definition of bioenergy type as the basis for differentiated approaches in the training of football players. Physical Culture: Upbringing, Education and Training, 2, 43-45.

Gibadullin, I. G. (2009). Structure of physical fitness and an integrated control system in the long-term preparation in biathlon: Monograph. Izhevsk: Izhevsk State Technical University.

Gizatullina, Ch. A. (2013). Functional training of athletes with different types of blood circulation and bioenergy (Doctoral Dissertation). Naberezhnye Chelny.

Green, R. (2006). Maximizing height, distance or rotation from real-time analysis visualisation of takeoff angles and speed. Journal of Sports Science and Medicine, 5, 590-596. PMCID: PMC3861760

Grigoryants, I. A. (2005). Artistic gymnastics: From Sydney to Athens. Theory and Practice of Physical Culture, 4, 28-34.
Kachouri, M., Vandewalle, H., \& Huet, M. (1996). Is the exhaustion time at maximal aerobic speed an index of aerobic endurance? Archives of Physiology and Biochemistry, 104(3), 330-336.

Karlenko, V. P., \& Karlenko, N. V. (2003). Using computer technology «D\&K-TEST» practice in training qualified athletes. physical culture and sport in contemporary socio-economic transformation in Russia: Materials jubilee scientific-practical. conference on the $70^{\text {th }}$ Anniversary VNIIFK. All-Russian Research Institute of Physical Culture and Sports (pp. 134-136).

Khalikov, G. Z. (2014). Preparation of athletes based on integrated functional assessment (Doctoral Dissertation). Naberezhnye Chelny.

Kirk Seeley, M., \& Bressel, E. (2005). A Comparison of upper-extremity reaction forces between the Yurchenko vault and floor exercise. Journal of Sports Science and Medicine, 4, 85-94.

Kozhevnikov, V. S. (2010). Differentiated approach to physical training of 15-year-old players, taking into account their bioenergy type. PedagogicalPsychological and Medico-Biological Problems of Physical Culture and Sports, 2(15), 41-48.

Kozhevnikov, V. S. (2011). Differentiated planning of fixed assets coaching soccer 15-16 years in the preparatory period on the basis of their bioenergy types (Doctoral Dissertation). Naberezhnye Chelny.

Kugaevsky, S. A. (2009). Using cardio-diagnostic D\&K-TEST for the individualization of training process short-trek qualifications. Physical Education Students, 2, 51-55.

Lebedev, N. I., \& Rozin, E. Y. (1981). Peculiarities of the physical development of promising gymnasts from 13 to 18 years old. Gymnastics, 1, 39-41.

Mahood, N. V., Kenefick, R. W., Kertzer, R., \& Quinn, T. J. (2001). Physiological determinants of cross- 
country ski racing performance. Medicine and Science in Sports and Exercise, 33(8), 1379-1384.

Marinšek, M., \& Čuk, I. (2010). Landing errors in the men's floor exercise are caused by flight characteristics. Biology of Sport, 27(2), 123-128. ICID: 913079.

Melikhova, T. M. (2007). Organizational-methodical bases and technologies of sports selection. Theory and Practice of Physical Culture, 4, 19-20.

Morozov, A. I. (2014). Exogenous respiratory interval hypoxic training in the preparation of middle distance runners (Doctoral dissertation). Naberezhnye Chelny.

Rozin, E. Y. (2001). Methodological aspects of the problem of selection and qualification system in particular. Physical Culture: Upbringing, Education and Training, 4, 2-6.

Rozin, E. Y., \& Mukambetov, A. A. (1980). Some morphofunctional peculiarities of children in terms of selection for artistic gymnastics. Gymnastics, 2, 15-18.
Sandbakk, O., Holmberg, H. C., \& Leirdal, S. (2010). Metabolic rate and gross efficiency at high work rates in world class and national level sprint skiers. European Journal of Applied Physiology, 109(3), 473-481. doi: 10.1123/IJSPP.2013-0383

Shlemin, A. M., \& Bril, M. S. (1978). Development of motor skills of young gymnasts. Gymnastics, 2, 12-16.

Smolevsky, V. M. (2005). Gymnastics: Evolution or type crisis? Theory and Practice of Physical Culture, 1, 30-32.

Vasilchuk, A. L., \& Gaverdovskiy, Y. K. (1985). Anthropometry indices of highly qualified female gymnasts as the factor of mastering the exercises. Gymnastics, 1, 8-13.

Wagner, P. D. (1996). Determinants of maximal oxygen transport and utilization. Annual Review of Physiology, $58,21-50$. 